\title{
Testing sterile neutrino mixing with present and future solar neutrino data
}

\author{
Kim Goldhagen ${ }^{1, \mathrm{a}}$, Michele Maltoni ${ }^{2, \mathrm{~b}}{ }^{\oplus}$, Shayne E. Reichard ${ }^{1, \mathrm{c}}$, Thomas Schwetz ${ }^{1, \mathrm{~d}}$ \\ ${ }^{1}$ Institut für Astroteilchenphysik, Karlsruher Institut für Technologie (KIT), Hermann-von-Helmholtz-Platz 1, 76344 Eggenstein-Leopoldshafen, \\ Germany \\ ${ }^{2}$ Instituto de Física Teórica UAM/CSIC, Universidad Autónoma de Madrid, Cantoblanco, Calle de Nicolás Cabrera 13-15, 28049 Madrid, Spain
}

Received: 6 October 2021 / Accepted: 25 January 2022 / Published online: 5 February 2022

(C) The Author(s) 2022

\begin{abstract}
We investigate the sensitivity of solar neutrino data to mixing of sterile neutrinos with masses $\gtrsim \mathrm{eV}$. For current data, we perform a Feldman-Cousins analysis to derive a robust limit on the sterile neutrino mixing. The solar neutrino limit excludes significant regions of the parameter space relevant to hints from reactor and radioactive gallium source experiments. We then study the sensitivity of upcoming solar neutrino data, most notably elastic neutrino-electron scattering in the DARWIN and DUNE experiments as well as coherent neutrino-nucleus scattering in DARWIN. These high precision measurements will increase the sensitivity to sterile neutrino mixing by about a factor of 4.5 compared to present limits. As a by-product, we introduce a simplified solar neutrino analysis using only four data points: the lowand high-energy $v_{e}$ survival and transition probabilities. We show that this simplified analysis is in excellent agreement with a full solar neutrino analysis; it is very easy to handle numerically and can be applied to any new physics model in which the energy dependence of the $v_{e}$ transition probabilities is not significantly modified.
\end{abstract}

\section{Contents}

1 Introduction . . . . . . . . . . . . . 1

2 Simplified solar neutrino analysis ........... 2

2.1 Probabilities . . . . . . . . . . . . 2

2.2 Simplified $\chi^{2}$ construction and comparison to full solar fit . . . . . . . . . . . 3

3 Monte Carlo analysis of present data $\ldots \ldots . .5$

\footnotetext{
a e-mail: kim.goldhagen@gmx.de

b e-mail: michele.maltoni@csic.es (corresponding author)

${ }^{c}$ e-mail: shayne.reichard@kit.edu

de-mail: schwetz@kit.edu
}

4 Sensitivity of future solar neutrino data to sterile neutrino mixing . . . . . . . . . 7

5 Summary and discussion . . . . . . . . . . . . 10 Appendix A: $\chi^{2}$ construction for Monte Carlo simulation 12 Appendix B: Details on the XENONnT/DARWIN anal-

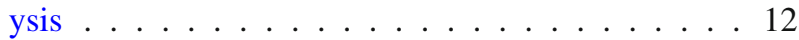

References . . . . . . . . . . . . . . 13

\section{Introduction}

Solar neutrinos have played a crucial role in the development of neutrino physics for many decades. In particular, they revealed the phenomenon of neutrino oscillations; see $[1,2]$ for reviews. In this work, we study some aspects of solar neutrinos in the context of sterile neutrinos. The hypothetical existence of light sterile neutrinos, with masses in the $\mathrm{eV}$ range, has been proposed in light of various experimental hints, which remain unexplained in terms of standard three-flavour oscillations; see e.g., [3,4] for recent reviews.

The effect of light sterile neutrinos on solar neutrinos has been studied by a number of authors (see, e.g., [5-11] for an incomplete list). Solar neutrinos provide a bound on the mixing of the heavy neutrino with the electron flavour, $\left|U_{e 4}\right|^{2}$, independent of the mass-squared difference $\Delta m_{41}^{2}$, as long as it is much larger than the one relevant to solar neutrino physics: $\Delta m_{41}^{2} \gg \Delta m_{21}^{2} \approx 7 \times 10^{-5} \mathrm{eV}^{2}$. Such a bound is especially relevant given various hints from reactor experiments [12-15] and radioactive source measurements in gallium experiments $[16,17]$. In particular, the claimed hint for sterile neutrino oscillations from the Neutrino-4 experiment [18] would require rather large values of $\left|U_{e 4}\right|^{2}$, which are in tension with the solar neutrino constraint; also see [19-21] for comments on Neutrino-4.

In this paper, we present a simplified solar neutrino analysis in which we cast a full fit to solar neutrino data [22], consisting of 303 data points, into just four effective data points 
that correspond to low- and high-energy $v_{e}$ survival and transition probabilities. We extract the corresponding observed values and correlation matrix from the full fit, allowing for a very efficient way to implement the information from solar neutrinos. This method can be applied to any new physics scenario that does not significantly modify the energy dependence of the $v_{e}$ transition probabilities. While the main application we have in mind here is the analysis of sterile neutrino constraints, the method is more general and may be applied to a wider class of new physics searches with solar neutrinos.

Our effective analysis allows one to capture the information from solar neutrinos in an accurate way that is based on quantities with clear and simple physics interpretation (high- and low-energy transition probabilities). Moreover, our method allows for a very numerically efficient implementation. As a first application, we perform a frequentist statistical analysis based on Monte Carlo simulation of the relevant test statistic in the sterile neutrino context and employing the Feldman-Cousins method [23]. In this way, we can evaluate the robustness of sterile neutrino constraints with respect to statistical properties of the analysis and derive solid frequentist confidence regions for the mixing angle $\theta_{12}$ and upper limits on $\sin ^{2} \theta_{14}=\left|U_{e 4}\right|^{2}$. While such analyses are typically computationally expensive, with the help of our implementation it can be performed within a few days on a single desktop computer.

As a second application of this method to treat solar neutrinos, we discuss how future solar neutrino observations will improve the sensitivity to sterile neutrino mixing. Current and future dark matter direct detection experiments [24-29] will offer highly precise measurements of solar neutrinos, either through Coherent Elastic Neutrino-Nucleus Scattering (CEvNS) or through Elastic Neutrino-Electron Scattering (EvES); see for example [30-32]. We focus here on the DARWIN project [28] and study its sensitivity to sterile neutrino mixing. The high precision determination of the low-energy $p p$ solar neutrino flux via $\mathrm{E} v \mathrm{ES}$ will provide especially valuable information in this respect. Furthermore, future neutrino detectors, such as DUNE [33] and HyperK [34] will offer very precise measurements of the high-energy ${ }^{8} \mathrm{~B}$ solar neutrino flux via EvES. As an example, we use the results of the detailed analysis performed in [35] for the DUNE liquid argon detector. We show that the high-energy measurements from DUNE and the low-energy measurements from DARWIN offer relevant complementarity and that the combination significantly improves the sensitivity to sterile neutrino mixing by roughly a factor 4.5 compared to current bounds. Furthermore, we discuss the complementarity with the precision determination of $\theta_{12}$ at the JUNO reactor experiment [36].

The outline of the paper is as follows: In Sect. 2, we introduce our four-data-point fit to solar neutrino data and define the simplified $\chi^{2}$ statistics. In Sect. 3, we present the results of our Monte Carlo simulation to determine frequentist confidence regions and limits for sterile neutrino mixing; whereas, in Sect. 4, we discuss the sensitivity of future data. A summary and discussion follow in Sect. 5. In Appendix A, we give technical details on the $\chi^{2}$ construction for the Monte Carlo simulation, and in Appendix B we provide details of our analysis of the $p p$ flux determination in xenon dark matter experiments.

\section{Simplified solar neutrino analysis}

\subsection{Probabilities}

We start by discussing the approximations adopted in the following to describe the relevant transition probabilities for solar neutrinos. The basic assumption is that neutrino evolution in the Sun is adiabatic and interference terms average out on the way from the Sun to the Earth, such that mass states arrive as an incoherent sum. This means the oscillation probabilities may be represented as:

$P_{e \alpha}=\sum_{k=1}^{4}\left|U_{e k}^{m}\right|^{2}\left|U_{\alpha k}\right|^{2}$,

where $U_{e k}^{m}$ is the effective mixing matrix element in matter at the production point inside the Sun, and we neglect the effects of Earth matter. We will consider four data points for our fit corresponding to the four oscillation probabilities:

$r=\left(P_{e e}^{L E}, P_{e e}^{H E}, P_{e x}^{L E}, P_{e x}^{H E}\right)$.

Here, $P_{e e}$ is the electron neutrino survival probability and $P_{e x}=P_{e \mu}+P_{e \tau}$ is the transition probability of electron neutrinos to the other active neutrino flavours. The indices $L E$ and $H E$ refer to low energy and high energy, where "low" and "high" mean below and above the MSW resonance [37,38]. Using (2.1), we find

$$
\begin{aligned}
& P_{e e}=\sum_{k=1}^{4}\left|U_{e k}^{m}\right|^{2}\left|U_{e k}\right|^{2}, \\
& P_{e x}=\sum_{k=1}^{4}\left|U_{e k}^{m}\right|^{2} \sum_{\alpha=\mu, \tau}\left|U_{\alpha k}\right|^{2}=1-P_{e e}-\sum_{k=1}^{4}\left|U_{e k}^{m}\right|^{2}\left|U_{s k}\right|^{2} .
\end{aligned}
$$

We parameterize the mixing matrix in terms of angles as in [10,39]:

$U=V_{34} V_{24} V_{14} V_{23} V_{13} V_{12}$,

where $V_{i j}$ is a rotation in the $i j$ plane with an angle $\theta_{i j}$, which in general can also contain a complex phase (see appendix $\mathrm{A}$ of [10] for a discussion). 
We now adopt the following approximations and assumptions: Due to NC matter effects as well as the NC measurement, solar neutrino data are in principle sensitive to $\theta_{34}$, $\theta_{24}, \theta_{23}[8,10]$, and generally complex phases may lead to physical effects in solar neutrinos $[10,11]$. However, fig. 6 of [39] shows the sensitivity of various datasets to the parameters $\left|U_{\mu 4}\right|=c_{14} s_{24}$ and $\left|U_{\tau 4}\right|=c_{14} c_{24} s_{34}$, with $s_{i j} \equiv \sin \theta_{i j}$ and $c_{i j} \equiv \cos \theta_{i j}$. From that plot, we can see that bounds from $v_{\mu}$ disappearance, atmospheric and long-baseline NC measurements are 1 to 2 orders of magnitude stronger than from solar data. Therefore, we conclude that once those bounds are taken into account, solar data is essentially independent of those parameters, and it should be a very good approximation to set them to zero. Therefore, we will set $\theta_{34}=\theta_{24}=0$ in our analysis. In this limit, we have for the relevant matrix elements:

$U=\left(\begin{array}{cccc}c_{12} c_{13} c_{14} & -s_{12} c_{13} c_{14} & -s_{13} c_{14} & -s_{14} \\ \cdot & \cdot & \cdot & 0 \\ \cdot & \cdot & \cdot & 0 \\ c_{12} c_{13} s_{14} & -s_{12} c_{13} s_{14} & -s_{13} s_{14} & c_{14}\end{array}\right)$.

Next, we consider matter effects in the Sun. We first take into account that $\left|\Delta m_{31}^{2}\right|, \Delta m_{41}^{2} \gg E_{v} V$ for relevant neutrino energies and matter potentials in the Sun, such that $\left|U_{e k}^{m}\right|^{2}=\left|U_{e k}\right|^{2}$ for $k=3,4$. This means that $\theta_{13}$ and $\theta_{14}$ are not unchanged by the matter effects. Second, we use the fact that for the energies relevant to the data points in our aforementioned analysis, we are either in the fully matter-dominated regime ("high energy") or in the vacuumdominated regime ("low energy"). Therefore, we set

$\theta_{12}^{m}=\theta_{12}$ (low energy), $\quad s_{12}^{m}=1$ (high energy)

We see that in our approximation the probabilities given in Eqs. (2.3) and (2.4) depend only on the three angles $\theta_{12}, \theta_{13}, \theta_{14}$ and are independent of complex phases. In [10], it has been shown, that the determination of $\theta_{13}$ is basically unaffected by the presence of a sterile neutrino. Therefore, we can use the constraint obtained from the 3-flavour fit. We have checked that varying $\theta_{13}$ within the uncertainty from present data has a negligible impact on our results. Therefore, we fix $s_{13}^{2}$ to the 3-neutrino best fit point $s_{13}^{2}=0.0223$, and we are left with the two parameters $s_{12}^{2}$ and $s_{14}^{2}$.

\subsection{Simplified $\chi^{2}$ construction and comparison to full solar fit}

The solar neutrino analysis used here is based on [22] and makes use of 303 data points in total. In particular, the data are: Chlorine total rate [40] (1 data point), Gallex and GNO total rates [41] (2 data points), SAGE total rate [42] (1 data point), SK1 full energy and zenith spectrum [43] (44 data points), SK2 full energy and day/night spectrum [44] (33 data points), SK3 full energy and day/night spectrum [45] (42 data points), SK4 2970-day day-night asymmetry [46] and energy spectrum [46] (24 data points), SNO combined analysis [47] (7 data points), Borexino Phase-I 741-day low-energy data [48] (33 data points), Borexino Phase-I 246-day high-energy data [49] (6 data points), Borexino Phase-II 408-day lowenergy data [50] (42 data points). The full solar neutrino $\chi^{2}$ includes various experimental and theoretical systematic uncertainties, encoded in 63 pull parameters, as well as the Standard Solar Model flux uncertainties [51].

In order to extract the information on the four observables from Eq. (2.2), we introduce an effective parameterization of the oscillation probabilities. We consider the exact oscillation probability at a reference point $P_{\text {ref }}(E)$. As a reference point, we choose the current 3 -flavour best fit point from the global analysis [22], which also determines the mass-squared difference $\Delta m_{21}^{2}$. The probability can refer either to the $e e$ or ex channel; we leave this point implicit to keep the notation simple. The two reference probabilities are shown in the topmiddle panel in Fig. 1. The low- and high-energy limits of that probability are denoted by $P_{\text {ref }}^{L E}$ and $P_{\text {ref }}^{H E}$, respectively:

$$
\begin{aligned}
& P_{\text {ref }}^{L E}=P_{\text {ref }}\left(E / E_{\text {res }} \rightarrow 0\right) \\
& P_{\text {ref }}^{H E}=P_{\text {ref }}\left(E / E_{\text {res }} \rightarrow \infty\right),
\end{aligned}
$$

where $E_{\text {res }} \approx 2 \mathrm{MeV}$ denotes the resonance energy. We then parameterize the shape of the probability by introducing the two parameters $P^{L E}$ and $P^{H E}$ in the following way:

$$
\begin{aligned}
P(E)= & {\left[P_{\mathrm{ref}}(E)-\frac{P_{\mathrm{ref}}^{L E}+P_{\mathrm{ref}}^{H E}}{2}\right] } \\
& \times \frac{P^{L E}-P^{H E}}{P_{\mathrm{ref}}^{L E}-P_{\mathrm{ref}}^{H E}}+\frac{P^{L E}+P^{H E}}{2} .
\end{aligned}
$$

This is simply a linear interpolation between the low and high energy values set by $P^{L E}$ and $P^{H E}$, while maintaining the shape in between the extreme values of the reference model $P_{\text {ref }}(E)$. In Fig. 1 we compare the parameterization from Eq. (2.9) with the shape obtained by numerical calculations of the probabilities without simplifying assumptions (dashed versus solid curves). We find good agreement within the relevant parameter range. We can now use this parameterization in the full solar neutrino analysis considering the four probabilities $P_{e e}^{L E}, P_{e e}^{H E}, P_{e x}^{L E}, P_{e x}^{H E}$ as fit parameters. For this purpose all relevant probabilities (e.g., for each solar neutrino flux, for day and night times, different detector locations) are parametrized as in Eq. (2.9). The results of this fit in terms of best fit values, standard deviations and correlation matrix are provided in Table 1 and shown for illustration also as data points with error bars in Fig. 1. The covariance matrix is obtained by calculating numerically the mixed second derivatives of the $\chi^{2}$ from the full fit at the $\chi^{2}$ minimum. 


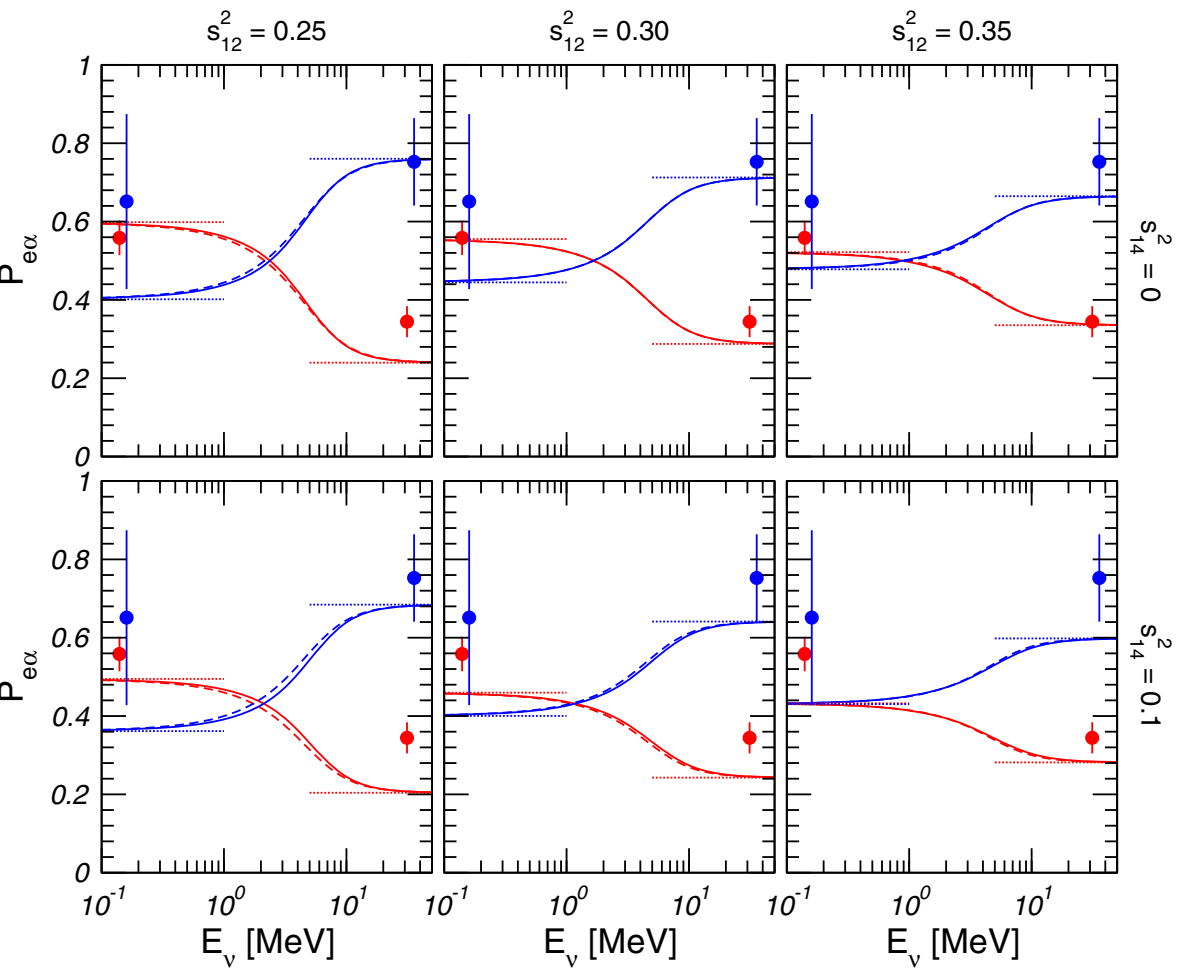

Fig. 1 Oscillation probabilities as a function of neutrino energy for different values of the mixing angles $s_{12}^{2}=0.25,0.3,0.35$ (columns) and $s_{14}^{2}=0,0.1$ (top and bottom rows, respectively), with $P_{e e}\left(P_{e x}\right)$ shown in red (blue). Solid curves correspond to the exact numerical probabilities, whereas dashed curves show our parameterization, Eq. (2.9). The middle panel in the top row corresponds to our reference point,
$P_{\text {ref }}(E)$, where solid and dashed curves are identical. Dotted horizontal lines indicate the asymptotic values $P^{L E}$ and $P^{H E}$. The data points with error bars show the observed values for the four probabilities, Eq. (2.2) as reported in Table 1 for the GS98 solar model (same in all panels). For definiteness we assume the ${ }^{8} \mathrm{~B}$ solar neutrino flux to average over the production region and we show day-time probabilities

Table 1 Best fit value for the observations $O_{r}$, their $1 \sigma$ uncertainties $\sigma_{r}$, and correlation matrix $\rho$ of the four observables. The upper (lower) part of the table corresponds to the GS98 (AGSS09) solar model [51]

\begin{tabular}{|c|c|c|c|c|c|c|}
\hline \multirow[b]{2}{*}{$P_{e e}^{L E}$} & \multirow{2}{*}{$\begin{array}{l}O_{r} \\
0.5585\end{array}$} & \multirow{2}{*}{$\begin{array}{l}\sigma_{r} \\
0.0440\end{array}$} & \multicolumn{4}{|c|}{ Correlation matrix } \\
\hline & & & +1.000 & +0.104 & -0.635 & +0.475 \\
\hline$P_{e e}^{H E}$ & 0.3444 & 0.0397 & +0.104 & +1.000 & +0.296 & +0.498 \\
\hline$P_{e x}^{L E}$ & 0.6512 & 0.2233 & -0.635 & +0.296 & +1.000 & -0.274 \\
\hline$P_{e x}^{H E}$ & 0.7526 & 0.1116 & +0.475 & +0.498 & -0.274 & +1.000 \\
\hline$\overline{P_{e e}^{L E}}$ & 0.5760 & 0.0441 & +1.000 & +0.087 & -0.636 & +0.448 \\
\hline$P_{e e}^{H E}$ & 0.3852 & 0.0424 & +0.087 & +1.000 & +0.297 & +0.515 \\
\hline$P_{e x}^{L E}$ & 0.6873 & 0.2277 & -0.636 & +0.297 & +1.000 & -0.250 \\
\hline$P_{e x}^{H E}$ & 0.8409 & 0.1179 & +0.448 & +0.515 & -0.250 & +1.000 \\
\hline
\end{tabular}

Qualitatively, the main contributions to our observables come from Gallium and Borexino for $P_{e e}^{L E}$, while other experiments contribute indirectly by constraining other solar neutrino flux contributions in Gallium experiments. The constraint on $P_{e x}^{L E}$ from current data is very poor and emerges only from the elastic electron scattering in Borexino. The $H E$ probabilities are constrained by SNO and SK. SNO NC data determines the total active neutrino flux, i.e., $P_{e e}^{H E}+P_{e x}^{H E}$, while SNO CC data constrain $P_{e e}^{H E}$. Elastic electron scattering in SK is sensitive to a certain combination of $P_{e e}^{H E}$ and $P_{e x}^{H E}$. Since the $H E^{8} \mathrm{~B}$ flux gives a sizeable contribution to the solar neutrino rate in Gallium experiments, a non-trivial correlation between $H E$ and $L E$ data points results.

Note that KamLAND reactor neutrino data [52] enter this analysis only indirectly through the determination of the value for $\Delta m_{21}^{2}$ used to calculate the reference probabili- 
ties $P_{\text {ref }}(E)$. Otherwise, our results do not depend on KamLAND data. In particular, we do not use information from KamLAND for our sterile neutrino limits, which would be subject to reactor neutrino flux uncertainties.

In order to derive a limit on $s_{14}^{2}=\left|U_{e 4}\right|^{2}$, we now build a $\chi^{2}$ function consisting of just four data points:

$\chi^{2}\left(s_{12}^{2}, s_{14}^{2}\right)=\sum_{r, s}\left(O_{r}-P_{r}\right) V_{r s}^{-1}\left(O_{s}-P_{s}\right)$.

The indices $r, s$ run over the four probabilities from Eq. (2.2), and $P_{r}\left(s_{12}^{2}, s_{14}^{2}\right)$ are the predicted values as discussed in the previous subsection. The "observed values" $O_{r}$ are the output of the full solar neutrino analysis described above and are given in Table 1. The covariance matrix $V$ in Eq. (2.10) is obtained in the following way. Let us define the relative covariance matrix for the observations as

$S_{r s}=\rho_{r s} \frac{\sigma_{r}}{O_{r}} \frac{\sigma_{s}}{O_{s}}$

(no sum over repeated indices), with $\rho_{r s}$ and $\sigma_{r}$ provided in Table 1. It turns out that a good approximation to the full solar neutrino fit is obtained by splitting the covariance matrix into an experimental and theoretical part as follows:

$V_{r s}=S_{r s}\left[\alpha O_{r} O_{s}+(1-\alpha) P_{r} P_{s}\right]$,

i.e., we assume that both the experimental and theoretical errors are proportional to $S_{r s}$ and that they have a relative weight set by the parameter $\alpha$. We find numerically that a value $\alpha \approx 0.35$ provides an excellent approximation. Let us note that Eq. (2.12) is purely a phenomenological ansatz in order to achieve a good match to the full numerical $\chi^{2}$ as shown in Fig. 2.

In Fig. 2, we compare the impact of the parameterization from Eq. (2.9), see black-dashed versus grey. Here, in both cases, the full $\chi^{2}$ statistical analysis from solar neutrino data is performed. The difference emerges only from the specific model adopted for the energy dependence of the oscillation probability. We see that the region is slightly shifted in $\sin ^{2} \theta_{12}$, whereas the approximation is excellent once we marginalize over $\sin ^{2} \theta_{12}$ and show the 1D $\Delta \chi^{2}$ curve in $\sin ^{2} \theta_{14}$. Finally, comparing the blue and black-dashed curves, we see that the 4-data point approach described above provides an excellent approximation to the full solar fit.

Our default SSM is the GS98 model. For comparison we show in the right panel of Fig. 2 also the 1D profile for the AGSS09 SSM. We find a slightly stronger constraint in that case, mostly due to the different predictions for the ${ }^{8} \mathrm{~B}$ flux.

Since our effective parameterization of $P(E)$ maintains the shape of the standard three-flavour probability to interpolate between the low- and high-energy regimes, our fit does not apply to new physics models that introduce a strong spectral distortion of the oscillation probability in the resonance region, such as non-standard neutrino interactions or sterile neutrinos with mass-squared differences comparable to $\Delta m_{21}^{2}$ (see [1] for a discussion). However, as long as the new physics scenario does not modify the energy dependence in the MSW resonance region and can be encoded in the limiting $H E$ and $L E$ probabilities, our analysis can be used as an effective way to take into account solar neutrino data. Apart from sterile neutrinos, another new-physics example to which our parameterization applies is generic non-unitarity in neutrino mixing, see e.g., [53] for a recent analysis including also solar neutrinos.

\section{Monte Carlo analysis of present data}

As a first application of the simplified solar neutrino analysis, we present a Monte Carlo simulation to determine the distribution of the $\Delta \chi^{2}$ test statistic used to construct confidence regions. The simplified analysis offers a very efficient calculation $^{1}$ of the $\Delta \chi^{2}$, which opens the possibility to perform a 2-dimensional Feldman-Cousins analysis of solar neutrino data within a few days on a single desktop computer.

First, we construct 2-dimensional confidence regions in the plane of $s_{12}^{2}$ and $s_{14}^{2}$. We consider the test statistic

$\Delta \chi^{2}\left(s_{12}^{2}, s_{14}^{2}\right)=\chi^{2}\left(s_{12}^{2}, s_{14}^{2}\right)-\chi_{\min }^{2}$,

where $\chi_{\min }^{2}$ is the minimum with respect to both parameters. We consider a 2-dimensional grid in $s_{12}^{2}$ and $s_{14}^{2}$ and at each grid point we generate $2.5 \times 10^{4}$ artificial Monte Carlo data sets $O_{r}^{\mathrm{MC}}$ for our four data points by assuming a multi-variate Gaussian distribution with mean $P_{r}\left(s_{12}^{2}, s_{14}^{2}\right)$ and covariance matrix $V_{r s}=S_{r s} P_{r} P_{s}$ and calculate $\Delta \chi^{2}$ with Eqs. (2.10) and (2.12) by replacing the true data with the generated pseudo-data: $O_{r} \rightarrow O_{r}^{\mathrm{MC}}$. In this way, we obtain the distribution of $\Delta \chi_{\mathrm{MC}}^{2}$ as a function of the true parameter values $\left(s_{12}^{2}, s_{14}^{2}\right)$. Then we can compare the $\Delta \chi^{2}$ value at each point for the actually observed data with the numerical distribution: the point $\left(s_{12}^{2}, s_{14}^{2}\right)$ is included in the $(1-\beta)$ CL interval if $\Delta \chi_{\text {observed }}^{2}$ is smaller than a fraction $\beta$ of the $\Delta \chi_{\mathrm{MC}}^{2}$ values at that parameter point.

The results of this analysis are shown in Fig. 3. We observe good agreement between the Monte Carlo results and the $\Delta \chi^{2}$ contours based on Wilks' theorem, for $68 \%$ and $90 \%$ CL, while some deviations become relevant at $99 \% \mathrm{CL}$ where the Monte Carlo regions are somewhat less constraining than the ones based on Wilks' theorem. This suggests that the tails of the distribution are somewhat larger than expected from the $\chi^{2}$ distribution.

Next, we want to consider 1-dimensional confidence intervals for a single parameter, irrespective of the other one. This

\footnotetext{
${ }^{1}$ See also Appendix A for comments on further computational speed up.
} 


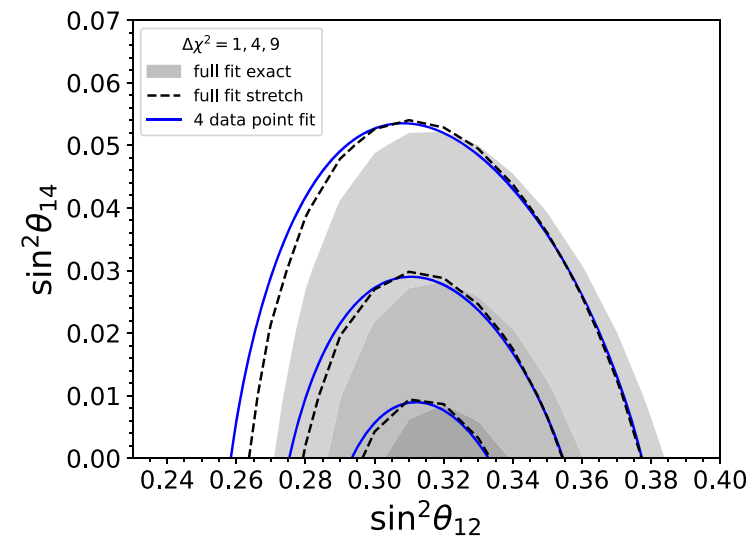

Fig. 2 Results for the GS98 solar models. We compare the exact solar neutrino fit (grey), the full solar fit but using the "stretch" parameterization for the probabilities from Eq. (2.9) (black-dash), and our 4-data

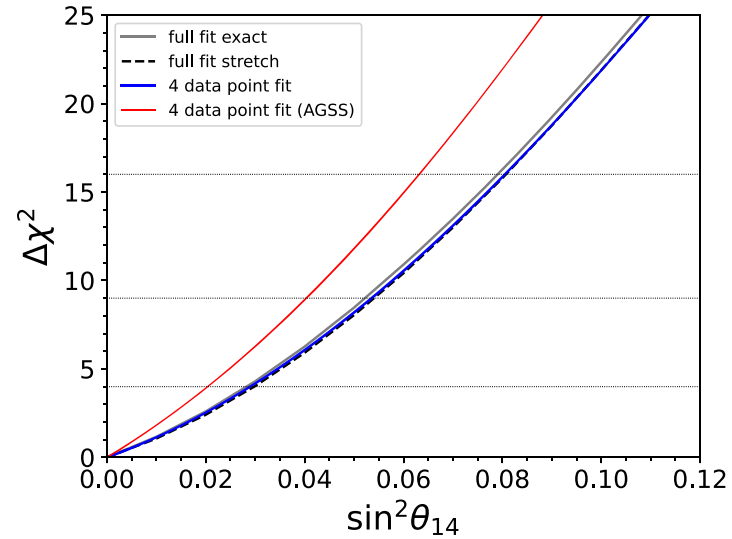

point approximation (blue). In the right panel we show in red the 4-data point fit for the AGSS09 solar model

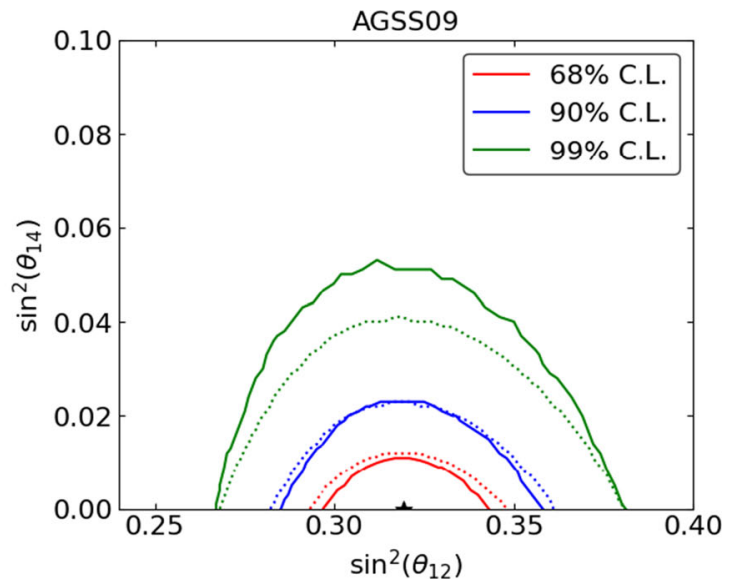

Fig. 3 Monte Carlo confidence regions at $68 \%, 90 \%$, and 99\% CL (solid) compared to $\Delta \chi^{2}$ contours based on Wilks' theorem using the $\chi^{2}$ distribution with 2 dof (dotted). The left (right) panel is for the GS98 (AGSS09) solar model

is a conceptually non-trivial problem because the distribution of the test statistic will in general depend on the unknown true value of the parameter over which to marginalized. For example, to derive confidence intervals for $s_{12}^{2}$, we consider the test statistic

$\Delta \chi^{2}\left(s_{12}^{2}\right)=\min _{s_{14}^{2}} \chi^{2}\left(s_{12}^{2}, s_{14}^{2}\right)-\chi_{\min }^{2}$.

The distribution of this quantity generally depends on the true value of $s_{14}^{2}$. Similarly, to derive confidence intervals for $s_{14}^{2}$ we consider the test statistic

$\Delta \chi^{2}\left(s_{14}^{2}\right)=\min _{s_{12}^{2}} \chi^{2}\left(s_{12}^{2}, s_{14}^{2}\right)-\chi_{\min }^{2}$,

whose distribution depends in general on the true value of $s_{12}^{2}$. In Fig. 4, we show the critical values for these test statistics as a function of the assumed true values by choosing the best fit point of the marginalized parameter as true value. By comparing the $\Delta \chi^{2}$ of the actually observed data with these critical values, confidence intervals can be constructed under the assumption of that particular true value of the marginalized parameter. The corresponding upper limits on $s_{14}$ are summarized in Table 2. The decrease of the critical values for $s_{14}^{2} \rightarrow 0$ can be attributed to the presence of the physical boundary $s_{14}^{2} \geq 0$, which leads to a decrease of the effective number of degrees of freedom. ${ }^{2}$ The impact of the physical boundary is even enhanced due to the fact that the fit actually pushes towards a best fit point out-side the physical boundary (especially for the AGSS09 model), c.f., Fig. 3. Note that the distribution of the test statistics, and therefore the critical values, are independent of the assumed solar model within our numerical accuracy. However, the resulting limits are notably stronger for the AGSS09 solar model.

A similar construction to that in Fig. 4 is carried out as a function of the assumed true value of the marginalized

\footnotetext{
${ }^{2}$ See [54] for a discussion of this effect.
} 


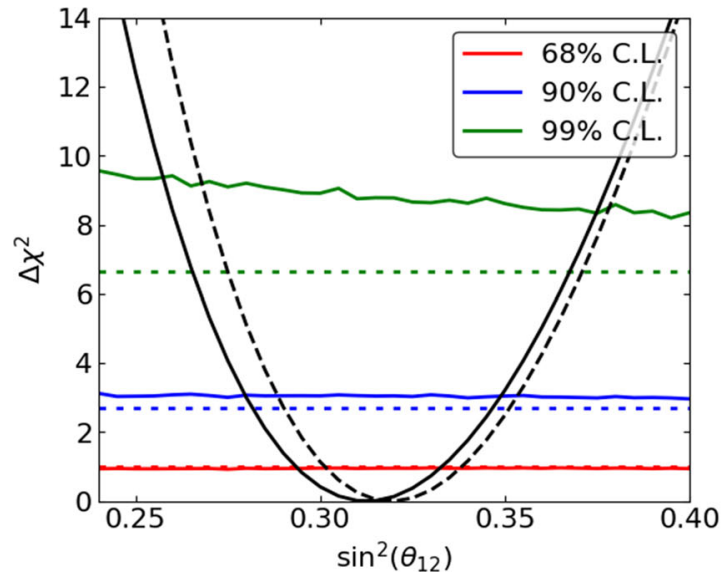

Fig. 4 Critical values of the test statistics Eqs. (3.2) and (3.3) for $68 \%, 90 \%$, and $99 \%$ CL (solid), assuming true values of $s_{14}^{2}=0$ and $s_{12}^{2}=0.313$, respectively. Dotted lines indicate the corresponding crit-

Table 2 Upper limits on $\sin ^{2} \theta_{14}$ at $90 \%$ and $99 \%$ CL from current solar neutrino data for the GS98 and AGSS09 solar models obtained with the MC simulation assuming a true value of $\sin ^{2} \theta_{12}=0.313$ (GS98) and 0.319 (AGSS09). The numbers in brackets would be the corresponding limits assuming a $\chi^{2}$-distribution with 1 dof for the test statistic

\begin{tabular}{lll}
\hline Solar model & $90 \%$ CL & $99 \%$ CL \\
\hline GS98 & $0.0168[0.0212]$ & $0.0446[0.0428]$ \\
AGSS09 & $0.0083[0.0145]$ & $0.0259[0.0314]$ \\
\hline
\end{tabular}

parameter. Then, we obtain confidence intervals for $s_{12}^{2}$ as a function of the true value of $s_{14}^{2}$, or upper limits on $s_{14}^{2}$ as a function of the true value of $s_{12}$, as shown in Fig. 5 . We observe that the confidence intervals for $s_{12}^{2}$ are basically independent of the true value of $s_{14}^{2}$ and are very close to the ones based on Wilks' theorem. Also the limits on $s_{14}^{2}$ are largely independent of the assumed true value for $s_{12}^{2}$. In this case, however, some deviations from limits based on Wilks theorem are visible, see also Table 2. The presence of the physical boundary, in particular, leads to somewhat stronger limits at low confidence level.

\section{Sensitivity of future solar neutrino data to sterile neutrino mixing}

As another application, we use the simplified analysis described in Sect. 2 to combine present solar data with possible future solar neutrino measurements and study their sensitivity to sterile neutrino mixing. The effective four-data point fit is easily extended to future experiments, as well. From the rich landscape of possible future solar neutrino measurements [32-34,55,56], we are going to consider the following exemplary data sets:

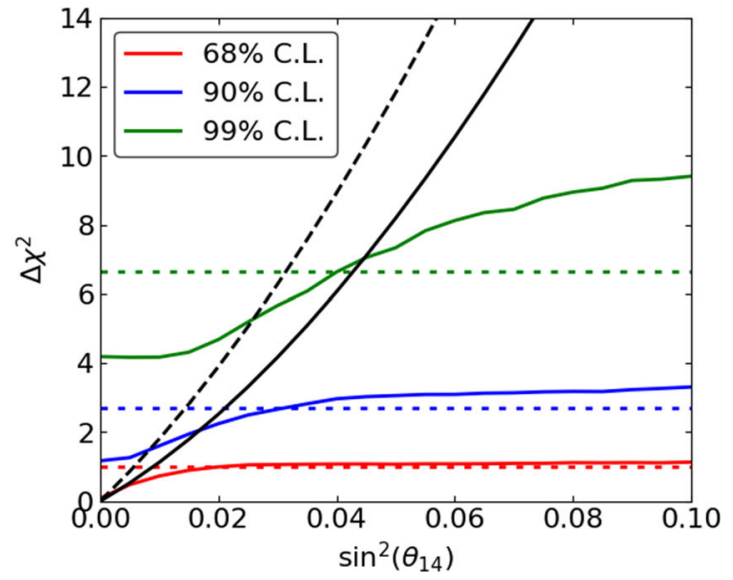

ical values of a $\chi^{2}$ distribution with 1 dof. The solid (dashed) black curves correspond to $\Delta \chi^{2}$ of the observed data for the GS98 (AGSS09) solar model

1. Elastic neutrino-electron scattering ( $E v E S)$ of lowenergy solar neutrinos in DARWIN and XENONnT. As discussed in [32], solar neutrinos in DARWIN offer a rich physics program. In particular, $\mathrm{E} v \mathrm{ES}$ events induced by the low-energy $p p$ solar neutrinos allow for a high precision determination of $P_{e e}^{L E}$ and $P_{e x}^{L E}$. We simulate electronic recoil events in the DARWIN detector, including various background components. The expected uncertainties on $P_{e e}^{L E}$ and $P_{e x}^{L E}$ and their correlation coefficient are extracted from a spectral fit to the simulated data (see Appendix B for details). Table 3 lists the relative precision and correlation values of the $p p$ flux components for selected exposures of the DARWIN detector. One of the dominant backgrounds for the $p p$ flux measurement is the radioactive isotope ${ }^{136} \mathrm{Xe}$ (see Fig. 8 in Appendix B). Therefore, in Table 3, we show the results both for the natural abundance of ${ }^{136} \mathrm{Xe}$ as well as the assumed depletion by two orders of magnitude. We see from the table that subpercent (percent-level) precision may be reached for a measurement of $P_{e e}\left(P_{e x}\right)$ in DARWIN. We do, however, note the strong correlation between $P_{e e}^{L E}$ and $P_{e x}^{L E}$, which is essentially independent of the exposure.

Indeed, we expect that $p p$ solar neutrino observations via $\mathrm{E} v \mathrm{ES}$ will soon become possible in the current generation of xenon dark matter experiments, XENONnT [24] and LZ [26]. In Table 4, we show the $p p$ flux sensitivity for selected exposures in XENONnT. The fiducial volume in XENONnT is smaller than that in DARWIN and, consequently, the materials background is higher, making the extraction of the $p p$ flux somewhat more difficult. In our XENONnT analysis, we assume the materials background presented in [57], which modeled a cryostat made of stainless steel rather than the titanium cryostat assumed for DARWIN. 

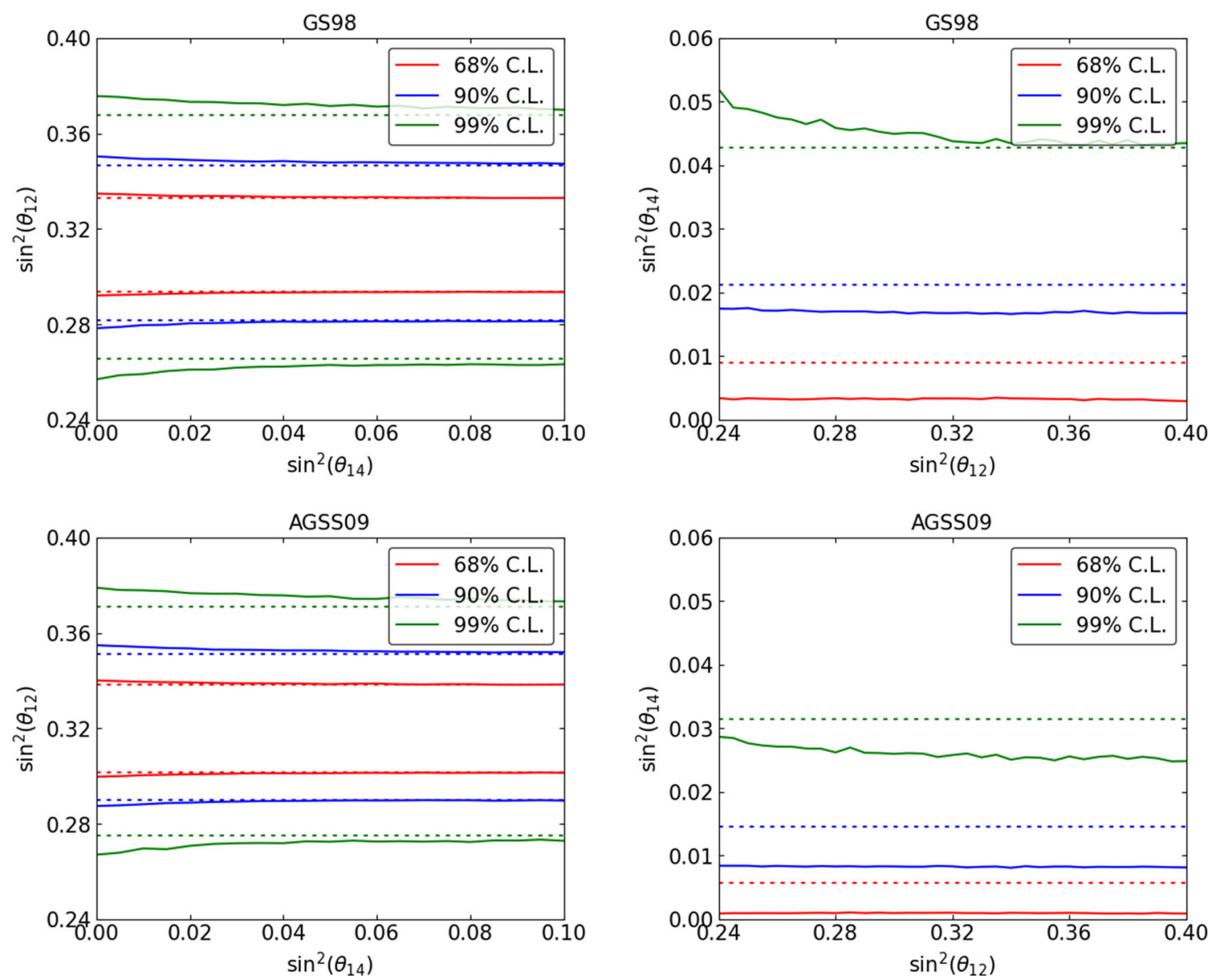

Fig. 5 Confidence interval at 68\%, 90\%, and 99\% CL for $s_{12}^{2}$ (left) and $s_{14}^{2}$ (right) as a function of the assumed true value of the other parameter, respectively. Dotted lines indicate the corresponding interval

assuming a $\chi^{2}$ distribution with 1 dof for the corresponding test statistic. The GS98 (AGSS09) solar model is adopted for the upper (lower) panels

Table 3 The relative uncertainties and variances of the $v_{e}$ and $v_{x}$ $(x=\mu, \tau)$ contributions to a $p p$ flux measurement in DARWIN and their correlation coefficient. Values are provided for five selected expo-

sures and two target compositions: natural ${ }^{136} \mathrm{Xe}$ abundance and depletion by two orders of magnitude. The fiducial mass of DARWIN is assumed to be around $30 \mathrm{t}$

\begin{tabular}{|c|c|c|c|c|c|}
\hline Exposure [ty] & $\sigma_{p p, e}[\%]$ & $\sigma_{p p, x}[\%]$ & $\rho_{e x}$ & $\sigma_{p p, e}^{2}$ & $\sigma_{p p, x}^{2}$ \\
\hline \multicolumn{6}{|l|}{ Natural } \\
\hline 1 & 12.9 & - & - & $1.67 \times 10^{-2}$ & - \\
\hline 30 & 1.1 & 5.3 & 0.979 & $1.14 \times 10^{-4}$ & $2.84 \times 10^{-3}$ \\
\hline 100 & 0.6 & 2.8 & 0.980 & $3.28 \times 10^{-5}$ & $8.11 \times 10^{-4}$ \\
\hline 300 & 0.3 & 1.6 & 0.980 & $1.09 \times 10^{-5}$ & $2.71 \times 10^{-4}$ \\
\hline 1000 & 0.2 & 0.9 & 0.980 & $3.23 \times 10^{-6}$ & $8.03 \times 10^{-5}$ \\
\hline \multicolumn{6}{|l|}{ Depleted } \\
\hline 1 & 6.4 & 25.0 & 0.986 & $4.07 \times 10^{-3}$ & $6.27 \times 10^{-2}$ \\
\hline 30 & 0.6 & 3.0 & 0.986 & $4.02 \times 10^{-5}$ & $8.75 \times 10^{-4}$ \\
\hline 100 & 0.4 & 1.6 & 0.986 & $1.23 \times 10^{-5}$ & $2.67 \times 10^{-4}$ \\
\hline 300 & 0.2 & 0.9 & 0.986 & $3.96 \times 10^{-6}$ & $8.60 \times 10^{-5}$ \\
\hline 1000 & 0.1 & 0.5 & 0.986 & $1.22 \times 10^{-6}$ & $2.65 \times 10^{-5}$ \\
\hline
\end{tabular}


Table 4 The relative uncertainties and variances of the flavor contributions to a $p p$ flux measurement in XENONnT are given with their corresponding correlation coefficient for three selected exposures. The fiducial mass of XENONnT is assumed to be around $4 \mathrm{t}$

\begin{tabular}{llllll}
\hline Exposure (ty) & $\sigma_{p p, e}(\%)$ & $\sigma_{p p, x}(\%)$ & $\rho_{e x}$ & $\sigma_{p p, e}^{2}$ & $\sigma_{p p, x}^{2}$ \\
\hline 1 & 15.2 & - & - & $2.32 \times 10^{-2}$ & - \\
5 & 3.8 & 17.2 & 0.977 & $1.45 \times 10^{-3}$ & $2.95 \times 10^{-2}$ \\
20 & 1.6 & 7.7 & 0.978 & $2.48 \times 10^{-4}$ & $6.00 \times 10^{-3}$ \\
\hline
\end{tabular}

2. Coherent neutrino-nucleus scattering (CEvNS) of highenergy solar neutrinos in DARWIN: DARWIN will also be able to observe CEvNS of ${ }^{8} \mathrm{~B}$ solar neutrinos, i.e., $\mathrm{NC}$ interactions, see [58] for a recent XENON analysis. This corresponds to a determination of $P_{e e}^{H E}+P_{e x}^{H E}$. We assume that this combination can be measured with a relative precision of $1 \%$, following the work of [59].

3. Elastic neutrino-electron scattering of high-energy solar neutrinos in DUNE: As detailed in [35], DUNE will be able to observe $\mathrm{CC}$ and elastic electron scattering of ${ }^{8} \mathrm{~B}$ solar neutrinos. From Fig. 3 of [35], we read off relative uncertainties of $0.4 \%$ for $P_{e e}^{H E}$ and $3.5 \%$ for $P_{e x}^{H E}$. We assume they are uncorrelated.

4. Determination of $\sin ^{2} \theta_{12}$ by JUNO reactor neutrinos: We also consider the very accurate determination of $\sin ^{2} \theta_{12}$ by the JUNO reactor experiment. ${ }^{3}$ Note that JUNO will have no sensitivity to $\sin ^{2} \theta_{14}$ for $\Delta m_{41}^{2} \gtrsim$ $0.1 \mathrm{eV}^{2}$ and therefore offers an independent determination of $\theta_{12}$. We are using the estimate of [55], Tab. 3-2, as the relative precision of $0.67 \%(1 \sigma)$ on $\sin ^{2} \theta_{12}$.

The future solar neutrino measurements are combined with current data by adding the future "measurements" as additional data points to the $\chi^{2}$ from present data, Eq. (2.10). The $4 \times 4$ correlation matrix from present data is extended correspondingly. In addition to the experimental errors quoted previously, we introduce fully correlated uncertainties of $0.6 \%$ on the $p p$ flux for the two $L E$ data points and $12 \%$ on the ${ }^{8} \mathrm{~B}$ flux for the $H E$ data points [51]. They are also correlated with present solar data. ${ }^{4}$ The JUNO measurement is added as a prior on $\sin ^{2} \theta_{12}$ without any further correlations. For the sensitivity estimate presented below we will continue to work under the assumption $\theta_{24}=\theta_{34}=0$. Note that our treatment of future data does not make use of the

\footnotetext{
3 JUNO will also be sensitive to solar neutrinos, see [55]. For instance, the ${ }^{8} \mathrm{~B}$ flux can be determined due to EvES, similar as in DUNE or HyperK. Here we use only the reactor information from JUNO.

${ }^{4}$ In order to correlate future data with current data one has to make sure that the resulting correlation matrix still leads to a valid covariance matrix with only positive eigenvalues. Note that the final fit is strongly dominated by future data; therefore, the precise way of how the correlation between present and future data is done has very little impact on the results.
}

parameterization Eq. (2.9); in each case outlined above the corresponding probabilities are determined independently.

The results are shown in Fig. 6 for various combinations of the aforementioned data sets. In all four panels, the grey region corresponds to current data, and the red region is the combination of current data with low-energy EvES data from DARWIN. We consider natural abundance of the ${ }^{136} \mathrm{Xe}$ isotope with a 100 ty exposure, corresponding to approximately 3 years of DARWIN data. Then, different combinations of DUNE, DARWIN high-energy CEvNS and JUNO are added.

While EvES data from DARWIN will make significant improvements over current data, a strong correlation between $s_{14}^{2}$ and $s_{12}^{2}$ limits the sensitivity. The correlation can be broken by complementary data, such as the high-precision determination of $s_{12}^{2}$ with JUNO (upper left panel of Fig. 6). The combination of DARWIN EvES $p p$ flux + DUNE $^{8} \mathrm{~B}$ (upper right) or DARWIN EvES $p p+\mathrm{CE} v \mathrm{NS}^{8} \mathrm{~B}$ (lower left) provide only modest improvements. However, the combination of all three solar observations shown in the lower right panel provide excellent sensitivity, comparable to the combination with JUNO. This is a consequence of the complementarity of the DUNE and DARWIN observations of the ${ }^{8} \mathrm{~B}$ flux, each providing strong sensitivity to a different combination of $P_{e e}^{H E}$ and $P_{e x}^{H E}$.

Figure 7 shows the $2 \sigma$ sensitivity to $\sin ^{2} \theta_{14}$ as a function of the XENONnT and DARWIN exposures for elastic neutrino-electron scattering (solid curves). The limit is obtained by searching for $\Delta \chi^{2}\left(\sin ^{2} \theta_{14}\right)=4$ and minimizing with respect to $\sin ^{2} \theta_{12}$. We observe that the somewhat higher background level in XENONnT leads only to a marginally worse sensitivity compared to DARWIN (natural ${ }^{136} \mathrm{Xe}$ ) at the same exposure, but the latter benefits of course from the larger attainable exposures. For the dashed curves, we assume additionally that $\sin ^{2} \theta_{12}$ is determined with a precision of $0.67 \%(1 \sigma)$ by JUNO. In this case, the ultimate sensitivity of $\sin ^{2} \theta_{14} \gtrsim 6.5 \times 10^{-3}$ is already achieved around 20 ty and is limited by the SSM $p p$ flux uncertainty. We see furthermore that in this case DARWIN will not be able to improve significantly beyond the sensitivity obtained after 5 years of XENONnT data. 

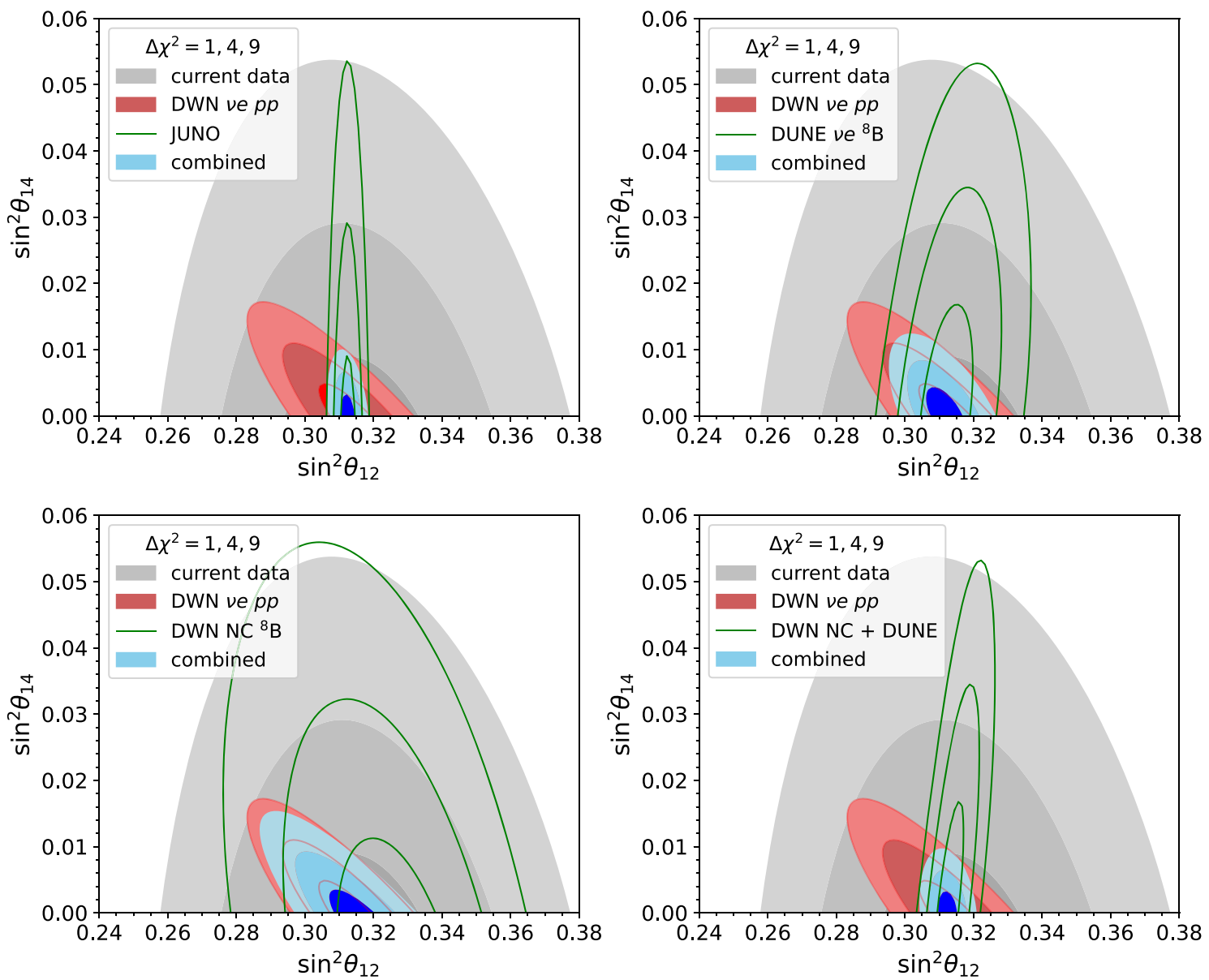

Fig. 6 Contours of $\Delta \chi^{2}=1,4,9$ in the plane of $\sin ^{2} \theta_{12}$ and $\sin ^{2} \theta_{14}$ for different combinations of current data (grey region) with future measurements. The red region in all panels corresponds to 100 ty exposure of DARWIN EvES data (natural ${ }^{136} \mathrm{Xe}$ abundance) combined with current

data. The green contours correspond to the indicated samples combined with current data. The blue region corresponds to the combination of all samples shown in their respective panel

\section{Summary and discussion}

In this paper, we have considered sterile neutrino mixing with the electron flavour, parametrized by $\sin ^{2} \theta_{14}=\left|U_{e 4}\right|^{2}$, in the context of solar neutrinos. The main results of this paper can be summarized as follows.

- We have presented a simplified solar neutrino analysis, which condenses a full-fledged solar neutrino fit into just four observables and their correlation matrix. The four observables are the $v_{e}$ survival probability and the $v_{e} \rightarrow$ $v_{\mu, \tau}$ transition probability, both at energies below and above the MSW resonance. These four probabilities have simple expressions in terms of the mixing parameters $\theta_{12}$ and $\theta_{14}$ and the resulting $\chi^{2}$ profile is an excellent approximation to the full solar neutrino fit. This analysis is insensitive to $\Delta m_{21}^{2}$, whose determination is dominated by KamLAND reactor neutrino data.

- We have performed a Feldman-Cousins (FC) analysis of present solar neutrino data, in terms of $\theta_{12}$ and $\theta_{14}$. We find that for the determination of $\theta_{12}$ the $\chi^{2}$ approximation is very well justified, and the result is basically independent of the presence of a sterile neutrino. However, we find some effects on $\theta_{14}$, where the FC analysis typically leads to stronger limits than the ones in the $\chi^{2}$ approximation. This can be traced back to the effect of the physical boundary $\sin ^{2} \theta_{14} \geq 0$, which leads to a decrease of the effective number of degrees of freedom. Furthermore, we find a rather strong dependence on the adopted solar model, where the $90 \%$ CL limit on $\sin ^{2} \theta_{14}$ differs by about a factor 2 between the GS 98 and AGSS09 solar models.

The current upper bounds on sterile neutrino mixing are summarized in Table 2 . These bounds are highly relevant to possible hints for sterile neutrinos from reactor experiments. In particular, the 90\% CL upper bound (GS98 


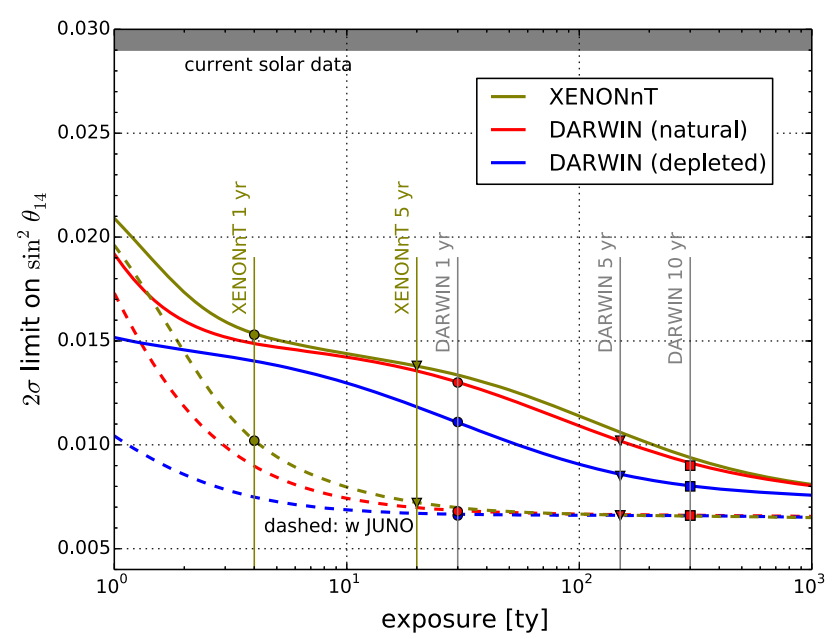

Fig. 7 Sensitivity to $\sin ^{2} \theta_{14}$ at $2 \sigma\left(\Delta \chi^{2}=4\right)$ from low-energy elastic neutrino-electron scattering in XENONnT and DARWIN as a function of the exposure, combined with current solar neutrino data (solid curves). For dashed curves we assume in addition that $\sin ^{2} \theta_{12}$ is determined with a precision of $0.67 \%(1 \sigma)$ by JUNO. For DARWIN we show in red the results for natural abundance of ${ }^{136} \mathrm{Xe}$, whereas blue curves assume depletion by two orders of magnitude. The grey shaded area indicates the region excluded by current solar neutrino data. Vertical lines indicate exposure times of 1,5 , and 10 years, assuming a fiducial mass of $4 \mathrm{t}$ for XENONnT and $30 \mathrm{t}$ for DARWIN

solar model) implies $\sin ^{2} 2 \theta_{14} \lesssim 0.07$, which is in conflict with the full $2 \sigma$ region reported by the Neutrino- 4 experiment [18]. A combined analysis of solar and reactor neutrino data is presented in [60], which provides a quantitative assessment of the impact of solar neutrino data on possible hints from reactor experiments.

- We have investigated the sensitivity of future solar neutrino measurements to sterile neutrino mixing. Elastic neutrino-electron scattering in the XENONnT and DARWIN dark matter experiments will provide a highprecision determination of the $p p$ solar neutrino flux; and elastic neutrino-electron scattering in DUNE and coherent neutrino-nucleus scattering in DARWIN will accurately measure the ${ }^{8} \mathrm{~B}$ solar neutrino flux. Additional complementary information comes from the JUNO reactor experiment, which will determine $\theta_{12}$ with subpercent precision. These data will have substantial sensitivity to sterile neutrino mixing, reaching $\sin ^{2} \theta_{14} \approx$ $6.5 \times 10^{-3}$, about a factor 4.5 better than the present limit and covering significant portions of the parameter space relevant to short-baseline reactor neutrino experiments. The ultimate sensitivity is limited by the uncertainty of the $p p$ solar neutrino flux prediction.

Throughout the paper we base our analysis on the four asymptotic (high and low energy) probabilities $P_{e e, e x}^{L E, H E}$. As we have demonstrated, this approach allows a very accurate description of present data in terms of sterile neutrino oscillations, after some tuning of analysis parameters. Furthermore, it allows for sensitivity forecasts of future data. However, once high-precision future data become available such an approach will need to be re-evaluated and eventually checked if relevant information is lost by considering only asymptotic probabilities.

Our method of fitting solar neutrino data can be applied to any new physics scenario that does not affect the spectral shape around the MSW resonance but only modifies the asymptotic values of the probabilities at low and/or high energies, such that the interpolation method described in Sect. 2 is accurate. This works very well for the sterile neutrino case considered here; another model which fulfills this requirement would be generic non-unitary mixing. For new-physics scenarios which modify transition probabilities in the MSW resonance region (such as for instance non-standard neutrino interactions or sterile neutrinos with mass-squared differences $\lesssim 10^{-5} \mathrm{eV}^{2}$ ) this approach would miss relevant effects and an analysis using explicitly information on the full solar neutrino energy is needed.

In conclusion, the results presented here demonstrate that solar neutrinos continue to provide relevant information on properties of neutrinos, and they will continue to do so for the foreseeable future.

Note added. After the completion of this work, the BEST collaboration released new results on radioactive source measurements in gallium [61], confirming the deficit reported by previous measurements $[16,17]$. The sterile neutrino mixing required to explain these results is in significant tension with the limit from solar neutrinos discussed here, see also $[60,62]$.

Acknowledgements This project has received support from the European Union's Horizon 2020 research and innovation programme under the Marie Sklodowska-Curie Grant agreement no. H2020-MSCAITN-2019-860881-HIDDeN, and by the Spanish grants PID2019110058GB-C21, SEV-2016-0597 and CEX2020-001007-S funded by MCIN/AEI/10.13039/501100011033.

Data Availability Statement This manuscript has no associated data or the data will not be deposited. [Authors' comment: All relevant data are given in the text and tables, in particular Tables. 1, 3 and 4.]

Open Access This article is licensed under a Creative Commons Attribution 4.0 International License, which permits use, sharing, adaptation, distribution and reproduction in any medium or format, as long as you give appropriate credit to the original author(s) and the source, provide a link to the Creative Commons licence, and indicate if changes were made. The images or other third party material in this article are included in the article's Creative Commons licence, unless indicated otherwise in a credit line to the material. If material is not included in the article's Creative Commons licence and your intended use is not permitted by statutory regulation or exceeds the permitted use, you will need to obtain permission directly from the copyright holder. To view a copy of this licence, visit http://creativecomm ons.org/licenses/by/4.0/.

Funded by $\mathrm{SCOAP}^{3}$. 


\section{Appendix A: $\chi^{2}$ construction for Monte Carlo simulation}

The covariance matrix defined in Eq. (2.12) depends on the parameters $\theta_{12}$ and $\theta_{14}$ via the predictions $P_{r}$. Therefore, $V_{r s}$ needs to be inverted numerically for each evaluation of the $\chi^{2}$ function when scanning over the parameters. In order to save the numerically expensive matrix inversion, we can split the inverse matrix $V^{-1}$ into experimental and theoretical uncertainties instead of $V$ :

$V_{r s}^{-1}=S_{r s}^{-1}\left[\alpha \frac{1}{O_{r}} \frac{1}{O_{s}}+(1-\alpha) \frac{1}{P_{r}} \frac{1}{P_{s}}\right]$

(no sum over repeated indices). It turns out that in this case $\alpha=0.5$ is a good choice. Since the matrix $S$ does not depend on the parameters, $S^{-1}$ needs to be calculated only once and for given $P_{r}$ we can directly construct $V^{-1}$ via Eq. (A.1). It can be shown that Eqs. (2.12) and (A.1) are equivalent up to linear order in $\epsilon_{r}=O_{r}-P_{r}$. We have checked that with the aforementioned adjustment of the coefficient $\alpha$ the two versions give numerically very similar results.

\section{Appendix B: Details on the XENONnT/DARWIN analy- sis}

We provide details of our analysis of solar-neutrino induced EvES events in the XENONnT and DARWIN dark matter experiments, following the work presented in [32].

Particles incident upon a dual-phase xenon Time Projection Chamber may scatter, or recoil, off a xenon nucleus (NR) or its electron cloud (ER). The most prominent sources of ER events arise from internal contaminants $\left({ }^{222} \mathrm{Rn},{ }^{85} \mathrm{Kr}\right)[63-$ 66], radioactive xenon isotopes $\left({ }^{136} \mathrm{Xe},{ }^{124} \mathrm{Xe}\right)$ [67-69], and the detector components themselves [67,70]. Imminently, the $p p$ solar neutrinos will constitute a comparable source of ER background events for dark matter searches in XENONnT and LZ; however, they will also unlock a novel science channel with unique probative value. DARWIN aims to reduce all other sources of ER events such that $p p$ solar neutrinos are the dominant (and irreducible) contributor.

The spectral fluxes of $p p,{ }^{13} \mathrm{~N}$, and ${ }^{15} \mathrm{O}$ neutrinos may be represented with the $\beta$ form,

$\frac{d \Phi_{i}}{d E_{v}}=\Phi_{i} A\left(x_{i}-E_{v}\right)\left[\left(x_{i}-E_{v}\right)^{2}-m_{e}^{2}\right]^{\frac{1}{2}} E_{v}^{2}$,

where $x_{i} \equiv Q_{i}+m_{e}, Q_{i}$ and $\Phi_{i}$ are the characteristic maximal energy and the flux scale of neutrino component $i$, respectively; $m_{e}$ is the electron mass, $A$ is the corresponding normalization factor, and $E_{v}$ is the energy of the emitted neutrino. For the $p p$ neutrinos, $\Phi_{p p}=5.98 \times 10^{10} \mathrm{~cm}^{-2} \mathrm{~s}^{-1}$ and $Q_{p p}=420 \mathrm{keV}$. In contrast, ${ }^{7} \mathrm{Be}$ and pep neutrinos are monoenergetic. The ${ }^{7} \mathrm{Be}$ neutrinos are emitted at $0.862 \mathrm{MeV}$ $(0.384 \mathrm{MeV})$ with a branching ratio of $90 \%(10 \%)$, while the pep neutrinos have an energy of $1.44 \mathrm{MeV}$. The flux scales are taken from the high-metallicity solar model [51].

These spectral fluxes are convolved with the differential cross section of elastic electron-neutrino scattering to determine the differential rate:

$\frac{d R_{i}}{d E_{r}}=N_{e} \sum_{\alpha} \int P_{e \alpha} \frac{d \Phi_{i}}{d E_{v}} \frac{d \sigma_{\alpha}}{d E_{r}} d E_{v}$,

where $P_{e \alpha}$ is the $v_{e} \rightarrow v_{\alpha}(\alpha=e, \mu, \tau)$ oscillation probability, $N_{e}=2.48 \times 10^{29}$ is the number of target electrons per tonne of xenon, and $E_{r}$ is the energy of the induced recoil. The differential E $v E S$ cross section is $[71,72]$

$\frac{d \sigma_{\alpha}}{d E_{r}}=\frac{2 G_{F}^{2} m_{e}}{\pi}\left[g_{L}^{2}+g_{R}^{2}\left(1-\frac{E_{r}}{E_{v}}\right)^{2}-g_{L} g_{R} \frac{m_{e} E_{r}}{E_{v}^{2}}\right]$.

For $\alpha=\mu, \tau$, the coupling constants are given by $g_{L}=$ $\sin ^{2} \theta_{w}-\frac{1}{2}$ and $g_{R}=\sin ^{2} \theta_{w}$, whereas for $v_{e}$ scattering $(\alpha=e), g_{L} \rightarrow g_{L}+1$ to account for its charged current interaction. A value of $\sin ^{2} \theta_{w}=0.2387$ [73] is assumed and kept fixed in the analysis. In order to induce an electronic recoil, an incident neutrino must possess more energy than the binding energy of a given shell; and, when a recoil occurs, its energy is lowered accordingly. For this reason, xenon is not completely sensitive to neutrinos with the lowest energies. This effect is incorporated in the neutrino scattering rates with a step function defined by the series of discrete electron binding energies from $12 \mathrm{eV}$ to $35 \mathrm{keV}$. This ultimately leads to a suppression of a few percent in the $p p$ neutrino event rate and negligible reductions for the other solar neutrino components. Furthermore, the Gaussian energy resolution obtained in XENON1T [74], which remain unchanged with the step approximation, is also applied:

$\frac{\sigma\left(E_{r}\right)}{E_{r}}=\frac{0.3171}{\sqrt{E_{r}[\mathrm{keV}]}}+0.0015$.

In order to include the information from DARWIN or XENONnT in the solar neutrino analysis described in the main text, we separate the terms of Eq. (B.2) by flavor for the $p p$ component. Namely, the contribution from electrontype neutrinos is written separately from muon- and tau-type neutrinos $(x=\mu, \tau)$ :

$$
\begin{aligned}
& \frac{d R_{p p}}{d E_{r}} \\
& =N_{e}\left[P_{e e} \int \frac{d \Phi_{p p}}{d E_{v}} \frac{d \sigma_{e}}{d E_{r}} d E_{v}+P_{e x} \int \frac{d \Phi_{p p}}{d E_{v}} \frac{d \sigma_{x}}{d E_{r}} d E_{v}\right] .
\end{aligned}
$$

Figure 8 shows the individual flavor contributions of the $p p$ recoil spectrum, along with ${ }^{7} \mathrm{Be}$ and the relevant ER backgrounds $[24,32,57,67]$. We perform a spectral fit to the data shown in the figure, leaving the normalizations of all the components as free fit parameters. For the $p p$ flux, we treat $P_{e e}$ 


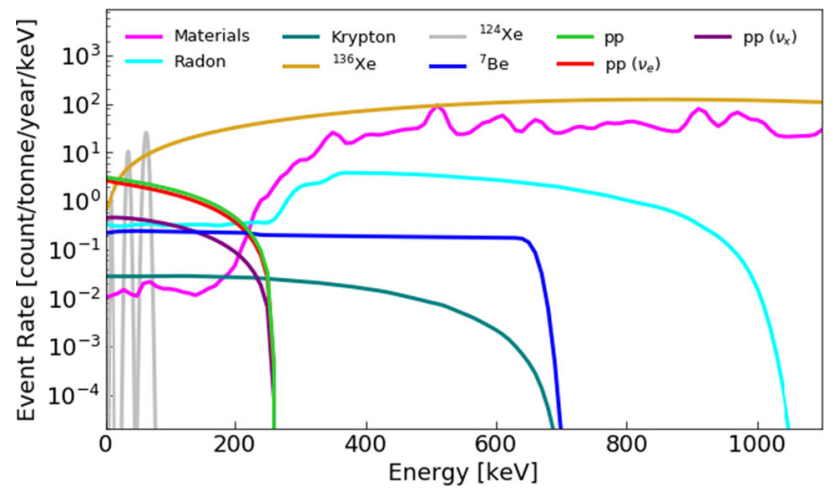

Fig. 8 The electronic recoil spectra of two solar neutrino components and five backgrounds up to $1.1 \mathrm{MeV}$. The $p p$ neutrino component is divided by lepton flavor. The solar components follow from the high-Z SSM model. The materials and ${ }^{136} \mathrm{Xe}$ events in $[1.1,3] \mathrm{MeV}$ (not shown) are also used in the statistical analysis. The materials component is based on a selection of events in a $30 \mathrm{t}$ fiducial volume

and $P_{e x}$ as free parameters (independent of energy), and we extract their covariance matrix by profiling over all the other ER components. Note that we also treat the ${ }^{7} \mathrm{Be}$ flux with free normalization, and therefore we can extract normalizations of the $p p$ flux components independent of a model, i.e., independent of the energy shape of the $v_{e}$ oscillation probabilities, as long as they are constant in the low-energy region relevant to $p p$ neutrinos.

\section{References}

1. M. Maltoni, A.Y. Smirnov, Solar neutrinos and neutrino physics. Eur. Phys. J. A 52(4), 87 (2016). https://doi.org/10.1140/epja/ i2016-16087-0 arXiv:1507.05287

2. G.D.O. Gann, K. Zuber, D. Bemmerer, A. Serenelli, The Future of Solar Neutrinos, Ann. Rev. Nucl. Part. Sci. 71, 491-528 (2021). arXiv:2107.08613

3. B. Dasgupta, J. Kopp, Sterile neutrinos. Phys. Rept. 928, 63 (2021). arXiv:2106.05913

4. S. Böser, C. Buck, C. Giunti, J. Lesgourgues, L. Ludhova et al., Status of light sterile neutrino searches. Prog. Part. Nucl. Phys. 111, 103736 (2020). https://doi.org/10.1016/j.ppnp.2019.103736 arXiv: 1906.01739

5. C. Giunti, M.C. Gonzalez-Garcia, C. Pena-Garay, Four-neutrino oscillation solutions of the solar neutrino problem. Phys. Rev. D 62, 013005 (2000). https://doi.org/10.1103/PhysRevD.62.013005 arXiv:hep-ph/0001101

6. M.C. Gonzalez-Garcia, M. Maltoni, C. Pena-Garay, Solar and atmospheric four neutrino oscillations. Phys. Rev. D 64, 093001 (2001). https://doi.org/10.1103/PhysRevD.64.093001 arXiv:hep-ph/0105269

7. J.N. Bahcall, M.C. Gonzalez-Garcia, C. Pena-Garay, If sterile neutrinos exist, how can one determine the total neutrino fluxes? Phys. Rev. C 66, 035802 (2002). https://doi.org/10.1103/PhysRevC.66. 035802 arXiv:hep-ph/0204194

8. C. Giunti, Y.F. Li, Matter effects in active-sterile solar neutrino oscillations. Phys. Rev. D 80, 113007 (2009). https://doi.org/10. 1103/PhysRevD.80.113007 arXiv:0910.5856
9. A. Palazzo, Testing the very-short-baseline neutrino anomalies at the solar sector. Phys. Rev. D 83, 113013 (2011). https://doi.org/ 10.1103/PhysRevD.83.113013 arXiv:1105.1705

10. J. Kopp, P.A.N. Machado, M. Maltoni, T. Schwetz, Sterile neutrino oscillations: the global picture. JHEP 05, 050 (2013). https://doi. org/10.1007/JHEP05(2013)050 arXiv:1303.3011

11. H.W. Long, Y.F. Li, C. Giunti, CP-violating phases in active-sterile solar neutrino oscillations. Phys. Rev. D 87(11), 113004 (2013). https://doi.org/10.1103/PhysRevD.87.113004 arXiv:1304.2207

12. G. Mention, M. Fechner, T. Lasserre, T.A. Mueller, D. Lhuillier et al., The reactor antineutrino anomaly. Phys. Rev. D 83, 073006 (2011). https://doi.org/10.1103/PhysRevD.83.073006 arXiv: 1101.2755

13. M. Dentler, A. Hernandez-Cabezudo, J. Kopp, M. Maltoni, T. Schwetz, Sterile neutrinos or flux uncertainties? - status of the reactor anti-neutrino anomaly. JHEP 11, 099 (2017). https://doi. org/10.1007/JHEP11(2017)099 arXiv:1709.04294

14. J.M. Berryman, P. Huber, Sterile neutrinos and the global reactor antineutrino dataset. JHEP 01, 167 (2021). arXiv:2005.01756

15. C. Giunti, Statistical significance of reactor antineutrino activesterile oscillations. Phys. Rev. D 101(9), 095025 (2020). https:// doi.org/10.1103/PhysRevD.101.095025 arXiv:2004.07577

16. C. Giunti, M. Laveder, Statistical significance of the gallium anomaly. Phys. Rev. C 83, 065504 (2011). https://doi.org/10.1103/ PhysRevC.83.065504 arXiv:1006.3244

17. J. Kostensalo, J. Suhonen, C. Giunti, P.C. Srivastava, The gallium anomaly revisited. Phys. Lett. B 795, 542-547 (2019). https://doi. org/10.1016/j.physletb.2019.06.057 arXiv:1906.10980

18. A. Serebrov et al., Search for sterile neutrinos with the Neutrino-4 experiment and measurement results. Phys. Rev. D 104(3), 032003 (2021). arXiv:2005.05301

19. PROSPECT, STEREO, M. Andriamirado et al., Note on arXiv:2005.05301, 'Preparation of the neutrino-4 experiment on search for sterile neutrino and the obtained results of measurements'. arXiv:2006.13147

20. P. Coloma, P. Huber, T. Schwetz, Statistical interpretation of sterile neutrino oscillation searches at reactors. Eur. Phys. J. C 81(1), 2 (2021). https://doi.org/10.1140/epjc/ s10052-020-08774-2 arXiv:2008.06083

21. C. Giunti, Y.F. Li, C.A. Ternes, Y.Y. Zhang, Neutrino4 anomaly: oscillations or fluctuations? Phys. Lett. B 816, 136214 (2021). https://doi.org/10.1016/j.physletb.2021.136214 arXiv:2101.06785

22. I. Esteban, M. Gonzalez-Garcia, M. Maltoni, T. Schwetz, A. Zhou, The fate of hints: updated global analysis of three-flavor neutrino oscillations. JHEP 09, 178 (2020). https://doi.org/10.1007/ JHEP09(2020)178 arXiv:2007.14792

23. G.J. Feldman, R.D. Cousins, A unified approach to the classical statistical analysis of small signals. Phys. Rev. D 57, 3873-3889 (1998). https://doi.org/10.1103/PhysRevD.57.3873 arXiv:physics/9711021

24. XENON, E. Aprile et al., Projected WIMP sensitivity of the XENONnT dark matter experiment. JCAP 11, 031 (2020). https:// doi.org/10.1088/1475-7516/2020/11/031. arXiv:2007.08796

25. PandaX-4T, Y. Meng et al., Dark matter search results from the PandaX-4T commissioning run. Phys. Rev. Lett. 127(26), 261802 (2021). arXiv:2107.13438

26. LZ, D.S. Akerib et al., The LUX-ZEPLIN (LZ) experiment. Nucl. Instrum. Methods A 953, 163047 (2020). https://doi.org/10.1016/ j.nima.2019.163047. arXiv:1910.09124

27. DarkSide, P. Agnes et al., DarkSide-50 532-day dark matter search with low-radioactivity argon. Phys. Rev. D 98(10), 102006 (2018). https://doi.org/10.1103/PhysRevD.98.102006. arXiv:1802.07198

28. DARWIN, J. Aalbers et al., Darwin: towards the ultimate dark matter detector. JCAP 1611, 017 (2016). https://doi.org/10.1088/ 1475-7516/2016/11/017. arXiv:1606.07001 
29. J. Billard et al., Direct detection of dark matter-APPEC committee report. arXiv:2104.07634

30. J. Billard, L.E. Strigari, E. Figueroa-Feliciano, Solar neutrino physics with low-threshold dark matter detectors. Phys. Rev. D 91(9), 095023 (2015). https://doi.org/10.1103/PhysRevD.91. 095023 arXiv: 1409.0050

31. D.G. Cerdeño, M. Fairbairn, T. Jubb, P.A.N. Machado, A.C. Vincent et al., Physics from solar neutrinos in dark matter direct detection experiments. JHEP 05, 118 (2016). https://doi.org/10.1007/ JHEP09(2016)048. arXiv:1604.01025. [Erratum: JHEP 09, 048 (2016)]

32. DARWIN, J. Aalbers et al., Solar neutrino detection sensitivity in DARWIN via electron scattering. Eur. Phys. J. C 80(12), 1133 (2020). https://doi.org/10.1140/epjc/s10052-020-08602-7. arXiv:2006.03114

33. DUNE, B. Abi et al., Deep underground neutrino experiment (DUNE), far detector technical design report, volume II: DUNE physics. arXiv:2002.03005

34. Hyper-Kamiokande, K. Abe et al., Hyper-Kamiokande design report. arXiv: 1805.04163

35. F. Capozzi, S.W. Li, G. Zhu, J.F. Beacom, DUNE as the nextgeneration solar neutrino experiment. Phys. Rev. Lett. 123(13), 131803 (2019). https://doi.org/10.1103/PhysRevLett.123.131803 arXiv: 1808.08232

36. JUNO, Z. Djurcic et al., Juno conceptual design report. arXiv: 1508.07166

37. L. Wolfenstein, Neutrino oscillations in matter. Phys. Rev. D 17, 2369-2374 (1978). https://doi.org/10.1103/PhysRevD.17.2369

38. S.P. Mikheev, A.Yu. Smirnov, Resonance amplification of oscillations in matter and spectroscopy of solar neutrinos. Sov. J. Nucl. Phys. 42, 913-917 (1985) [Yad. Fiz. 42, 1441 (1985)]

39. M. Dentler, A. Hernandez-Cabezudo, J. Kopp, P.A.N. Machado, M. Maltoni et al., Updated global analysis of neutrino oscillations in the presence of eV-scale sterile neutrinos. JHEP 08, 010 (2018). https://doi.org/10.1007/JHEP08(2018)010 arXiv:1803.10661

40. B.T. Cleveland, T. Daily, R. Davis Jr., J.R. Distel, K. Lande et al., Measurement of the solar electron neutrino flux with the homestake CHLorine detector. Astrophys. J. 496, 505-526 (1998). https://doi. org/10.1086/305343

41. F. Kaether, W. Hampel, G. Heusser, J. Kiko, T. Kirsten, Reanalysis of the Gallex Solar neutrino flux and source experiments. Phys. Lett. B 685, 47-54 (2010). https://doi.org/10.1016/j.physletb. 2010.01.030 arXiv:1001.2731

42. SAGE, J.N. Abdurashitov et al., Measurement of the solar neutrino capture rate with gallium metal. III: Results for the 2002-2007 datataking period. Phys. Rev. C 80, 015807 (2009). https://doi.org/10. 1103/PhysRevC.80.015807. arXiv:0901.2200

43. Super-Kamiokande, J. Hosaka et al., Solar neutrino measurements in super-Kamiokande-I. Phys. Rev. D 73, 112001 (2006). https:// doi.org/10.1103/PhysRevD.73.112001. arXiv:hep-ex/0508053

44. Super-Kamiokande, J.P. Cravens et al., Solar neutrino measurements in super-Kamiokande-II. Phys. Rev. D 78, 032002 (2008). https://doi.org/10.1103/PhysRevD.78.032002. arXiv:0803.4312

45. Super-Kamiokande, K. Abe et al., Solar neutrino results in superKamiokande-III. Phys. Rev. D 83, 052010 (2011). https://doi.org/ 10.1103/PhysRevD.83.052010. arXiv:1010.0118

46. Y. Nakajima, in "SuperKamiokande." Talk given at the XXIX International Conference on Neutrino Physics and Astrophysics, Chicago, USA, June 22-July 2, 2020 (online conference)

47. SNO, B. Aharmim et al., Combined analysis of all three phases of solar neutrino data from the Sudbury Neutrino Observatory. Phys. Rev. C 88, 025501 (2013). https://doi.org/10.1103/PhysRevC.88. 025501. arXiv: 1109.0763

48. G. Bellini et al., Precision measurement of the $7 \mathrm{Be}$ solar neutrino interaction rate in Borexino. Phys. Rev. Lett. 107,
141302 (2011). https://doi.org/10.1103/PhysRevLett.107.141302 arXiv: 1104.1816

49. Borexino, G. Bellini et al., Measurement of the solar 8B neutrino rate with a liquid scintillator target and $3 \mathrm{MeV}$ energy threshold in the Borexino detector. Phys. Rev. D 82, 033006 (2010). https:// doi.org/10.1103/PhysRevD.82.033006. arXiv:0808.2868

50. BOREXINO, G. Bellini et al., Neutrinos from the primary protonproton fusion process in the Sun. Nature 512(7515), 383-386 (2014). https://doi.org/10.1038/nature13702

51. N. Vinyoles, A.M. Serenelli, F.L. Villante, S. Basu, J. Bergström et al., A new generation of standard solar models. Astrophys. J. 835(2), 202 (2017). https://doi.org/10.3847/1538-4357/835/2/202 arXiv: 1611.09867

52. KamLAND, A. Gando et al., Reactor on-off antineutrino measurement with KamLAND. Phys. Rev. D 88(3), 033001 (2013). https:// doi.org/10.1103/PhysRevD.88.033001. arXiv:1303.4667

53. S.A.R. Ellis, K.J. Kelly, S.W. Li, Current and future neutrino oscillation constraints on leptonic unitarity. JHEP 12, 068 (2020). https://doi.org/10.1007/JHEP12(2020)068 arXiv:2008.01088

54. J. Elevant, T. Schwetz, On the determination of the leptonic CP phase. JHEP 09, 016 (2015). https://doi.org/10.1007/ JHEP09(2015)016 arXiv:1506.07685

55. JUNO, F. An et al., Neutrino physics with JUNO. J. Phys. G 43(3), 030401 (2016). https://doi.org/10.1088/0954-3899/43/3/030401. arXiv: 1507.05613

56. Jinping, J.F. Beacom et al., Physics prospects of the Jinping neutrino experiment. Chin. Phys. C 41(2), 023002 (2017). https://doi. org/10.1088/1674-1137/41/2/023002. arXiv:1602.01733

57. XENON, E. Aprile et al., Physics reach of the Xenon1T dark matter experiment. JCAP 04, 027 (2016). https://doi.org/10.1088/ 1475-7516/2016/04/027. arXiv: 1512.07501

58. XENON, E. Aprile et al., Search for coherent elastic scattering of solar ${ }^{8} \mathrm{~B}$ neutrinos in the XENON1T dark matter experiment. Phys. Rev. Lett. 126, 091301 (2021). https://doi.org/10.1103/ PhysRevLett.126.091301. arXiv:2012.02846

59. J. Ekert, Calculation of the coherent elastic neutrino-nucleus scattering of solar ${ }^{8} \mathrm{~B}$ neutrinos in DARWIN. B.Sc. thesis, Karlsruhe Institute of Technology (2021)

60. J.M. Berryman, P. Coloma, P. Huber, T. Schwetz, A. Zhou, Statistical significance of the sterile-neutrino hypothesis in the context of reactor and gallium data. arXiv:2111.12530

61. V.V. Barinov, et al., Results from the Baksan experiment on sterile transitions (BEST). arXiv:2109.11482

62. V. Barinov, D. Gorbunov, BEST impact on sterile neutrino hypothesis. arXiv:2109.14654

63. XENON100, E. Aprile et al., Online ${ }^{222} \mathrm{Rn}$ removal by cryogenic distillation in the XENON100 experiment. Eur. Phys. J. C 77(6), 358 (2017). https://doi.org/10.1140/epjc/ s10052-017-4902-x. arXiv:1702.06942

64. M. Murra, Intrinsic background reduction by cryogenic distillation for the XENON1T darkmatter experiment. PhD thesis, WWU Münster (2019)

65. XENON, E. Aprile et al., Xenon1T dark matter data analysis: signal and background models and statistical inference. Phys. Rev. D 99(11), 112009 (2019). https://doi.org/10.1103/PhysRevD.99. 112009. arXiv:1902.11297

66. XENON, E. Aprile et al., Removing krypton from Xenon by cryogenic distillation to the PPq level. Eur. Phys. J. C 77(5), 275 (2017). https://doi.org/10.1140/epjc/s10052-017-4757-1. arXiv: 1612.04284

67. DARWIN, F. Agostini et al., Sensitivity of the DARWIN observatory to the neutrinoless double beta decay of ${ }^{136} \mathrm{Xe}$. Eur. Phys. J. C 80(9), 808 (2020). https://doi.org/10.1140/epjc/ s10052-020-8196-z. arXiv:2003.13407

68. C. Wittweg, B. Lenardo, A. Fieguth, C. Weinheimer, Detection prospects for the second-order weak decays of ${ }^{124} \mathrm{Xe}$ in multi- 
tonne xenon time projection chambers. Eur. Phys. J. C 80(12), 1161 (2020). https://doi.org/10.1140/epjc/s10052-020-08726-w arXiv:2002.04239

69. XENON, E. Aprile et al., Observation of two-neutrino double electron capture in ${ }^{124} \mathrm{Xe}$ with XENON1T. Nature 568(7753), 532-535 (2019). https://doi.org/10.1038/s41586-019-1124-4. arXiv: 1904.11002

70. XENON, E. Aprile et al., Material radioassay and selection for the Xenon1T dark matter experiment. Eur. Phys. J. C 77(12), 890 (2017). https://doi.org/10.1140/epjc/s10052-017-5329-0. arXiv: 1705.01828

71. W.J. Marciano, Z. Parsa, Neutrino electron scattering theory. J. Phys. G 29, 2629-2645 (2003). https://doi.org/10.1088/ 0954-3899/29/11/013 arXiv:hep-ph/0403168
72. J.A. Formaggio, G.P. Zeller, From eV to Eev: neutrino cross sections across energy scales. Rev. Mod. Phys. 84, 1307-1341 (2012). https://doi.org/10.1103/RevModPhys.84.1307 arXiv:1305.7513

73. J. Erler, M.J. Ramsey-Musolf, The weak mixing angle at low energies. Phys. Rev. D 72, 073003 (2005). https://doi.org/10.1103/ PhysRevD.72.073003 arXiv:hep-ph/0409169

74. XENON, E. Aprile et al., Energy resolution and linearity of Xenon1T in the MeV energy range. Eur. Phys. J. C 80(8), 785 (2020). https://doi.org/10.1140/epjc/s10052-020-8284-0. arXiv:2003.03825 